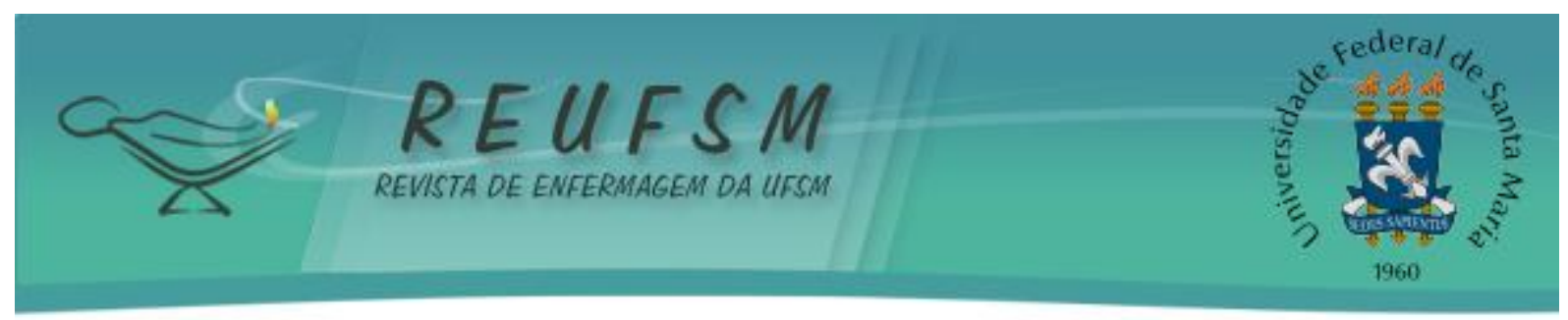

ARTIGO ORIGINAL

\title{
ESCALA DE AVALIAÇÃO DA DOR: PERCEPÇÃO DOS ENFERMEIROS EM UMA UNIDADE DE TERAPIA INTENSIVA NEONATAL
}

\author{
SCALE OF PAIN EVALUATION: PERCEPTION OF NURSES IN A NEONATAL INTENSIVE CARE \\ UNIT
}

ESCALA DE EVALUACIÓN DEL DOLOR: PERCEPCIÓN DE LOS ENFERMEROS EN UNA UNIDAD DE TERAPIA INTENSIVA NEONATAL

\author{
Xênia Martins Monfrim ${ }^{1}$ \\ Larissa de Aguiar Saraiva \\ Cristiane Lima de Moraes ${ }^{3}$ \\ Aline da Costa Viegas ${ }^{4}$
}

\section{Doi: $10.5902 / 2179769215049$}

RESUMO: Objetivo: conhecer a percepção de enfermeiros com relação à utilização de um instrumento para avaliação da dor em neonatos prematuros. Método: pesquisa qualitativa, exploratória e descritiva, realizada em uma Unidade de Terapia Intensiva Pediátrica e Neonatal, de um Hospital Universitário de Pelotas, Rio Grande do Sul. Participaram do estudo quatro enfermeiros, no período de outubro e novembro de 2010. Os dados foram coletados por meio de entrevista semiestruturada e analisados de acordo com a análise temática. Resultados: foram abordadas as seguintes categorias: Escala para avaliação da dor nos RNs prematuros: percepção dos enfermeiros e Escala de dor e o prematuro: sua implementação. Conclusão: enfermeiros desconhecem as novas tecnologias utilizadas para mensurar a dor; contudo, todos se mostraram comprometidos com uma assistência humanizada, uma vez que relataram interesse pela inserção deste instrumento de avaliação.

Descritores: Dor; Prematuro; Unidades de Terapia Intensiva Neonatal; Escalas; Enfermagem.

ABSTRACT: Aim: to identify the perceptions of nurses in relation to the use of an instrument to assess pain in preterm infants. Method: qualitative, exploratory and descriptive research conducted in a Neonatal Intensive Care Unit, in a University Hospital in Pelotas, Rio Grande do Sul. The study included four nurses, in the period of October to November 2010. Data were collected through semi-structured interviews and analyzed according to thematic analysis. Results: the following categories were addressed: Scale to assess pain in premature newborns: perception of nurses, and Pain Scale and premature newborns: implementation. Conclusion: nurses do not know the new technologies used to

\footnotetext{
Recorte do Trabalho de Conclusão de Curso “Avaliação da Dor em RN's prematuros: Percepção dos Enfermeiros numa Unidade de Terapia Intensiva Neonatal”, apresentado à Faculdade de Enfermagem da Universidade Católica de Pelotas (UCPel), em 2010.

1 Enfermeira. Mestre em Saúde e Comportamento. Enfermeira assistencial do Hospital Universitário São Francisco de Paula e da Prefeitura Municipal de Pelotas. Pelotas, Rio Grande do Sul, Brasil. E-mail: xenia.monfrim@bol.com.br

${ }^{2}$ Enfermeira. Enfermeira do Núcleo de Desenvolvimento Humano da Unimed Pelotas. Pelotas, Rio Grande do Sul, Brasil. E-mail: lalasaraiva@gmail.com

${ }^{3}$ Enfermagem. Mestre em Enfermagem. Docente da Universidade Católica de Pelotas. Pelotas, Rio Grande do Sul, Brasil. E-mail: krismoraes31@hotmail.com

${ }^{4}$ Enfermeira. Mestre em Ciências. Doutoranda pelo Programa de Pós Graduação da Faculdade de Enfermagem da Universidade Federal de Pelotas. Bolsista de Demanda Social CAPES. Pelotas, Rio Grande do Sul, Brasil. Email: alinecviegas@hotmail.com
} 


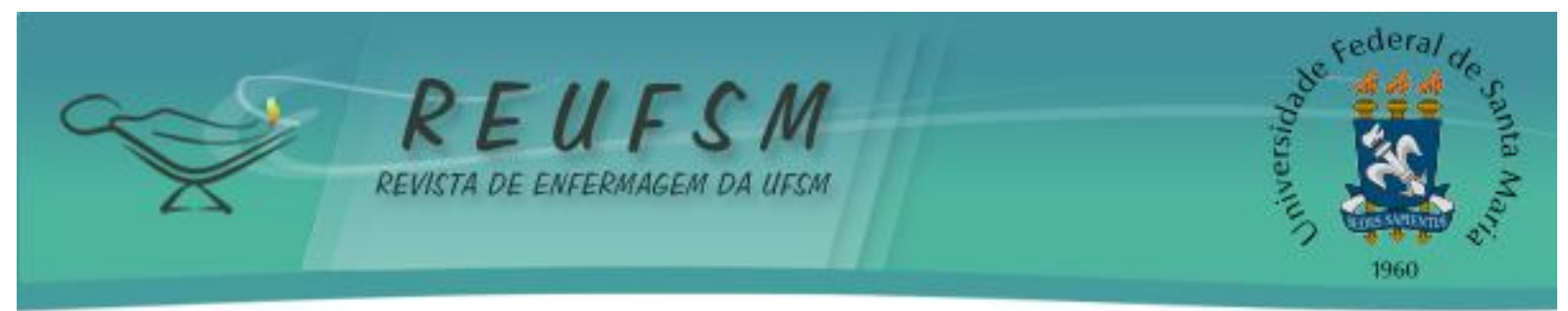

measure pain; however, all nurses demonstrated committment to a humanized assistance, as they reported being interested in the inclusion of this instrument of assessment.

Descriptors: Pain; Infant Premature; Intensive Care Units; Neonatal; Scales; Nursing.

RESUMEN: Objetivo: investigar la percepción entre los enfermeros sobre el uso de un instrumento para evaluar el dolor en recién nacidos prematuros. Método: investigación cualitativa, exploratoria y descriptiva, realizada en una Unidad de Cuidados Intensivos Pediátricos y Neonatales del Hospital de Pelotas (RS, Brasil). Cuatro enfermeras participaron en el estudio, entre octubre y noviembre de 2010. Los datos fueron recogidos por entrevistas semi-estructurada y analizados según el análisis temático. Resultados: se estudiaron las siguientes categorías: Escala para evaluar el dolor en los recién nacidos prematuros: percepción de los enfermeros y La escala de dolor y el prematuro: su aplicación. Conclusión: los enfermeros desconocen las nuevas tecnologías utilizadas para medir el dolor, sin embargo, todos estaban comprometidos con una atención humanizada, mostrando interés por la inserción de este instrumento de evaluación.

Descriptores: Dolor; Prematuro; Unidades de Cuidado Intensivo Neonatal; Escalas; Enfermería.

\section{INTRODUÇÃO}

Todos os anos nascem no mundo em média 20 milhões de recém-nascidos (RNs) de baixo peso, muitos deles em virtude de partos prematuros. Este fato corrobora de forma expressiva com a elevação das taxas de mortalidade neonatal. ${ }^{1}$

Contudo, os avanços na neonatologia tanto do ponto de vista tecnológico, bem como da veiculação de evidências científicas tem acarretado em grandes melhorias no cuidado dos RNs prematuros. Dessa forma, a inevitável internação nas Unidades de Terapia Intensiva Neonatal (UTIN) para alguns prematuros, mostra-se imprescindível para excluir e/ ou amenizar fatores de risco emergente do quadro clínico em que se encontram.

Quando da necessidade de internação nas UTIN, os RNs prematuros ficam sujeitos a diversas técnicas e procedimentos invasivos e potencialmente dolorosos, os quais acarretam na diminuição de sua qualidade de vida e desenvolvimento neuropsicomotor. ${ }^{2}$ Tais tratamentos mantêm os bebês vivos, contudo ocasionam, por vezes, dor e sofrimento. ${ }^{3}$

A dor é caracterizada por sua complexidade, subjetividade e multidimensionalidade, sendo assim, o alívio desta e a promoção de conforto são medidas indispensáveis, as quais envolvem conhecimento científico, habilidade técnica, além de questões humanitárias e éticas da prática de enfermagem. ${ }^{4}$

Nesse interim, a exposição ao stress e a dor neonatal também estão associados com o desenvolvimento cerebral alterado. Destarte, é de extrema relevância que sejam identificados com precisão e de forma adequada. ${ }^{5}$

Para qualificar e quantificar a dor, normalmente, faz-se uso de instrumentos que utilizam parâmetros comportamentais e fisiológicos. Existe, atualmente, uma variedade de instrumentos desenvolvidos para decodificar a linguagem da dor sentida pelos RNs. ${ }^{6}$ Dentre esses instrumentos cita-se as escalas para avaliação da dor.

Considerou-se a Escala da Dor, Agitação e Sedação do Neonato (N- PASS) a mais apropriada para este estudo, visto que essa avalia diversos estágios de comportamento dos RNs relacionados à dor. ${ }^{7}$ Pode-se observar que existe um grande número de instrumentos publicados e já validados no Brasil e no mundo o que refletem a dificuldade de se avaliar de forma adequada e precisa a dor. ${ }^{8}$ Salienta-se que independentemente da escala escolhida para avaliação da dor, esta verificação deve ser realizada regularmente, de forma sistemática, devendo ser considerada como quinto sinal vital. ${ }^{3}$ 


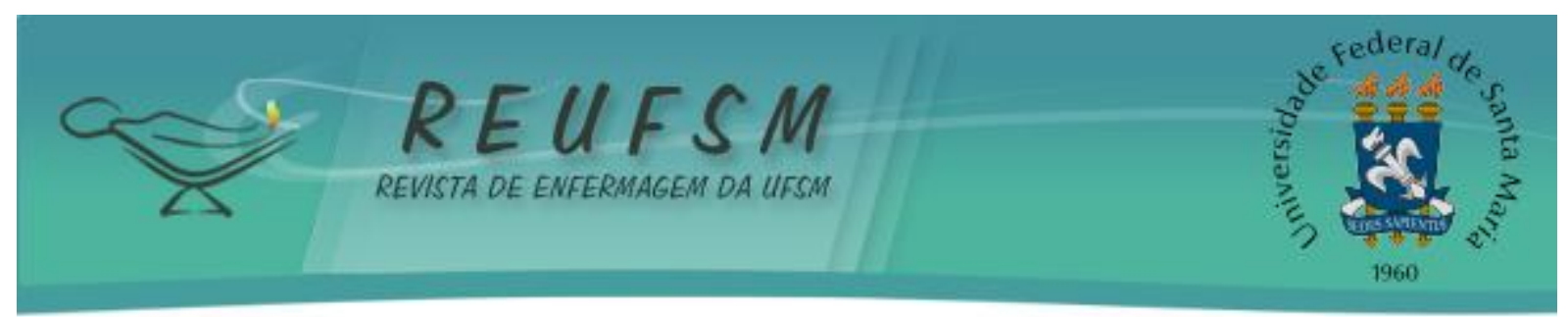

Nesse contexto, a enfermagem desempenha papel fundamental, uma vez que cuidar pressupõe, também, estar atento a subjetividade do paciente, de modo a intervir no curso dos sintomas, dentre eles a dor, permitindo conforto e bem-estar. Dessa maneira, na medida em que se mensura a dor como sinal vital, tem-se parâmetros para estabelecer um adequado plano de cuidados. Assim, considerando que o cuidado terapêutico necessita estar condicionado à intensidade da dor, especialmente os enfermeiros, devem ter competências e habilidades para avaliá-la, e logo, implementar estratégias de alívio da mesma e monitorar a eficácia das intervenções. ${ }^{9}$

Para tanto, a utilização destas escalas está relacionada com ações de prevenção e controle da dor, as quais são de grande importância para os neonatos, pois podem evitar efeitos deletérios a curto e longo prazo, desencadeados a partir da estimulação dolorosa. ${ }^{10}$

Assim sendo, acredita-se que por meio da sensibilização dos enfermeiros das UTIN e pediátrica quanto à pertinência da avaliação da dor nos prematuros e a conscientização sobre a importância da utilização das escalas, esta prática poderá ser incorporada na rotina de enfermagem.

Nessa perspectiva, a questão que norteou o estudo foi: qual a percepção de enfermeiros atuantes em uma Unidade de Terapia Intensiva Pediátrica e Neonatal, com relação à utilização de um instrumento para avaliação da dor em neonatos prematuros?

0 objetivo deste artigo foi conhecer a percepção de enfermeiros com relação à utilização de um instrumento para avaliação da dor em neonatos prematuros.

\section{MÉTODO}

Trata-se de um estudo exploratório, descritivo, com abordagem qualitativa. A pesquisa foi realizada com quatro enfermeiros que prestavam assistência a RNs prematuros, em uma UTIN e pediátrica, de um Hospital Universitário, localizado na cidade de Pelotas, Rio Grande do Sul, nos meses de outubro e novembro de 2010.

Essa unidade conta com 10 leitos, sendo um deles de isolamento. Observa-se que a maior frequência de internações são de pacientes prematuros. Ainda, no período trabalhavam cinco enfermeiros, distribuídos nos turnos manhã, tarde, noite um e dois, e o designado para realizar as folgas.

Os critérios de inclusão utilizados foram: estar atuando como enfermeiro na UTIN e pediátrica; ter disponibilidade para participar da sensibilização em relação ao estudo; ter disponibilidade para aplicar o instrumento de avaliação da dor nos recém-nascidos prematuros; autorizar a gravação da entrevista; permitir a divulgação dos dados em meio científico. 0 critério de exclusão foi: estar atuando há menos de seis meses na unidade, visto há necessidade do profissional ter experiência na mesma.

Os métodos utilizados para coleta de dados foram: Escala da Dor, Agitação e Sedação no Neonato (N-PASS) ${ }^{7}$, aplicada pelos enfermeiros, em todos os prematuros hospitalizados nesse período, a qual inclui critérios de avaliação como choro, comportamento, expressão facial, tono nas extremidades e sinais vitais.

Destaca-se que, nesta etapa da pesquisa, foi solicitado que os enfermeiros aplicassem a escala durante o período de duas semanas aos prematuros internados, de modo que obtivessem uma aproximação e sensibilização com o instrumento, já que essa não era utilizada na unidade. Para tanto, pontua-se que não foi dado continuidade a utilização da mesma pela instituição.

Ainda, foi aplicada entrevista semiestruturada, composta por sete questões abertas, realizada com esses profissionais no mês seguinte à aplicação da escala, a qual foi gravada e transcrita na sua íntegra. As questões englobavam aspectos relacionados ao conhecimento prévio de escalas para avaliação da dor, a percepção e viabilidade de 


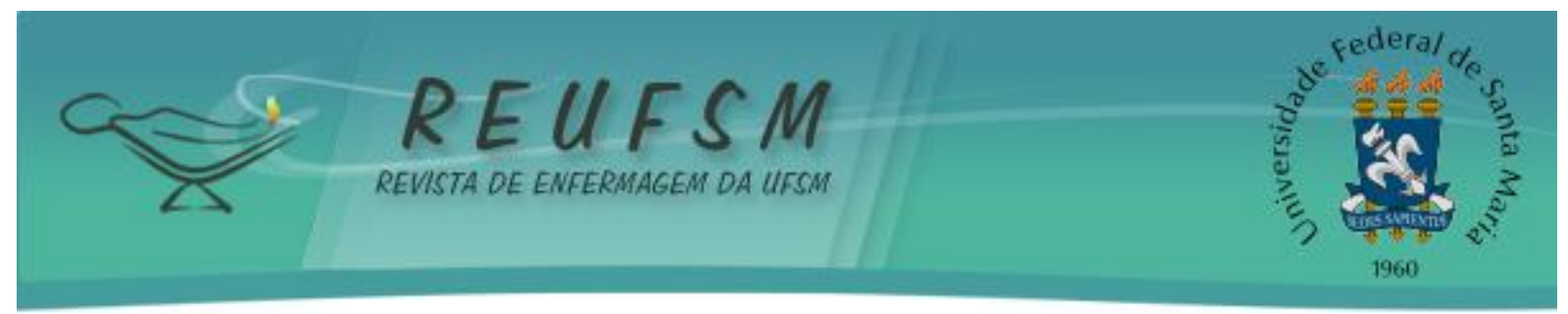

aplicação do instrumento, benefícios, facilidades e dificuldades da escala, e por último o desejo de utilizá-la na prática profissional.

Com o intuito de facilitar a condução da pesquisa, antes da coleta dos dados foi realizada uma sensibilização e treinamento dos enfermeiros, por meio de orientações técnicas e teóricas, objetivando informá-los sobre a utilização da Escala para Avaliação da Dor nos RNs Prematuros. Dos cinco profissionais sensibilizados, quatro deles aderiram ao estudo.

Para garantir os princípios éticos da pesquisa, aos participantes foram encaminhadas cartas apresentando o objetivo, bem como os convidando para participarem. Após o aceite, foi solicitado que assinassem o termo de consentimento livre e esclarecido, em duas vias. Para resguardar a identidade dos participantes e manter a privacidade, eles foram identificados por nomes de pedras preciosas, conforme a escolha dos mesmos.

O percurso metodológico teve início após a obtenção do consentimento institucional, para a realização da pesquisa no hospital escolhido; em posse deste, o projeto foi encaminhado ao Comitê de Ética em Pesquisa de uma Universidade local, no qual foi apreciado e aprovado de acordo com o parecer 2010/26. Este estudo respeitou as diretrizes sobre pesquisa com seres humanos, foi aprovado na vigência da Resolução 196/96, mas que está de acordo e não fere os princípios da Resolução vigente, qual seja, a 466/12 do Conselho Nacional de Saúde. ${ }^{11}$

Os dados que emergiram deste estudo foram analisados de acordo com a análise temática de Minayo. ${ }^{12}$ A ordenação, classificação e análise final dos dados possibilitou a definição de duas categorias, denominadas: Escala para avaliação da dor nos RNs prematuros: percepção dos enfermeiros e Escala de dor e o prematuro: sua implementação, as quais contemplaram subcategorias.

\section{RESULTADOS E DISCUSSÃO}

Inicialmente, serão apresentadas as características dos enfermeiros. Participaram do estudo três mulheres e um homem, com idade compreendida dos 24 aos 36 anos. Em relação à escolaridade, todos apresentavam graduação em enfermagem e não possuíam pós graduação. № que diz respeito ao vínculo empregatício todos eram vinculados ao regime de Consolidação das Leis do Trabalho. A seguir serão apresentados os resultados oriundos da pesquisa.

\section{Escala para avaliação da dor nos RNs prematuros: percepção dos enfermeiros}

Essa categoria contempla as subcategorias: Conhecimento e viabilidade da aplicação do instrumento para avaliação da dor nos RNs prematuros.

Com relação ao conhecimento dos enfermeiros relacionado às escalas para avaliação da dor nos RNs prematuros, percebeu-se que todos estes não conheciam nenhum tipo de instrumento que proporcionasse este tipo de avaliação, anteriormente a realização deste estudo.

No que diz respeito, a viabilidade da aplicação do instrumento para avaliação da dor nos RNs prematuros, alguns consideraram favoráveis a sua aplicação.

\section{Viabilidade de $100 \%$ porque todos os pacientes precisam de acompanhamento e analgesia para a dor. (Brilhante) \\ [...] viabilidade é a analgesia, é o uso dela para analgesia das crianças, principalmente nas sedações. (Topázio)}

Nessas falas, destacam-se a importância da utilização de escalas para avaliar a dor nos recém-nascidos e o uso da analgesia. Desta forma, pode-se observar que a utilização da escala traz benefícios ao RN, desde que seja acompanhada de métodos que minimizem 


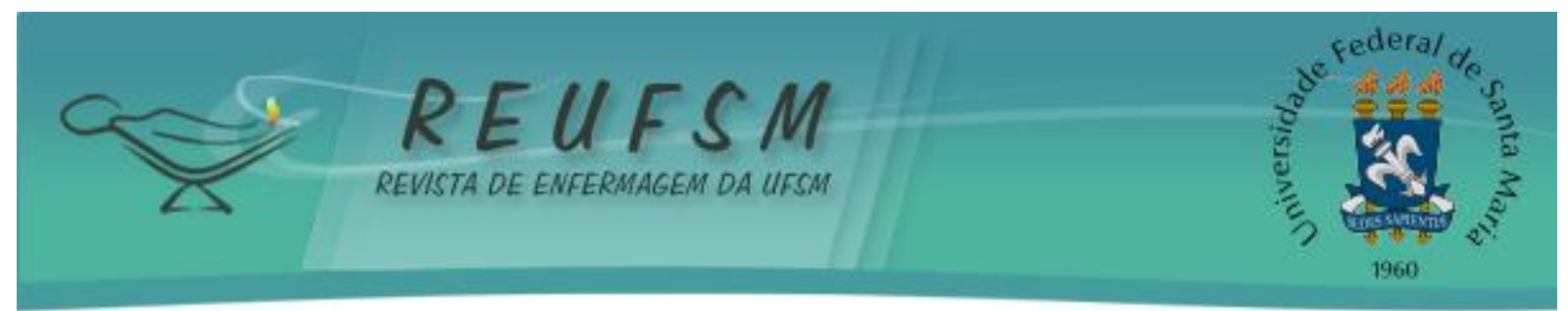

ou extinguem a dor daqueles que não sabem verbalizá-la, permitindo que terapêuticas medicamentosas sejam utilizadas apenas quando, evidentemente, imprescindíveis. Nesse sentido, avaliar a dor significa buscar dados acurados para a determinação de ações, que possam aliviá-la ou aboli-la. ${ }^{13}$

Para outros participantes, o "talvez" permaneceu como uma inconstância na viabilidade deste instrumento, ficando atrelada a elementos do serviço, tais como o aumento no quadro de funcionários ou acadêmicos de enfermagem, e capacitação profissional para avaliação dos sinais de dor no prematuro, como explicitado nas falas:

[...] disponibilidade de acadêmicos ou mais um enfermeiro de oito horas [...] (Rubi)

[...] instrumento que tem uma grande importância, desde que seja aplicado de forma coerente [...] porque no recém-nascido é difícil da gente interpretar sinais de dor [...] (Pérola)

Neste contexto, percebe-se que os profissionais, na maioria das vezes, apesar de reconhecerem a importância da utilização de escalas para avaliação da dor não a utilizam. Em um estudo desenvolvido com 15 enfermeiros em uma unidade neonatal da cidade de Maceió, Alagoas, apenas uma enfermeira afirmou utilizar escala para avaliação da dor no $\mathrm{RN}$, sendo que as demais, realizavam observação individualizada e critérios particulares, sem serem padronizados, os quais dificultam uma terapêutica adequada. ${ }^{14}$

Acredita-se que este fato encontra-se atrelado à formação acadêmica, visto que ainda existem muitos cursos que não priorizam essa temática no cuidado à criança, mais especificamente à avaliação da dor no RN prematuro. Contudo, acredita-se que, se houver maior investimento na capacitação e sensibilização dos enfermeiros, haverá possibilidade do desenvolvimento de um trabalho eficaz no cuidado integral desta criança.

\section{Escala de dor e o prematuro: sua implementação}

A análise dessa categoria permitiu a ordenação de duas subcategorias: facilidades e/ou benefícios e dificuldades vivenciadas pelos enfermeiros na aplicação de um instrumento para avaliação da dor nos RNs prematuros.

As facilidades e/ou benefícios vivenciados pelos enfermeiros na aplicação de um instrumento para avaliação da dor nos prematuros, estavam relacionadas ao registro, conhecimento/aprimoramento, avaliação, analgesia e participação da equipe. Quanto ao registro das informações obtidas na avaliação da dor, um dos participantes salienta em sua fala:

Importante para ter registro, uma quantificação [...] hoje a gente avalia a dor empiricamente [...] (Rubi)

Acredita-se que, no cuidado ao RN enfermo, os registros individualizados de cada profissional são de suma importância, uma vez que essa ação busca contribuir para a integralidade da assistência prestada pela equipe multiprofissional. Diante disso, torna-se possível utilizar métodos específicos para alívio da dor e, consequentemente, minimizar os agravos que podem estar associados.

Um estudo apontou que se a dor não for gerenciada adequadamente, pode haver consequências substanciais sobre o cérebro e os sistemas de estresse em desenvolvimento de recém-nascidos prematuros, sendo o manejo da dor considerado ainda um desafio. ${ }^{5}$ 


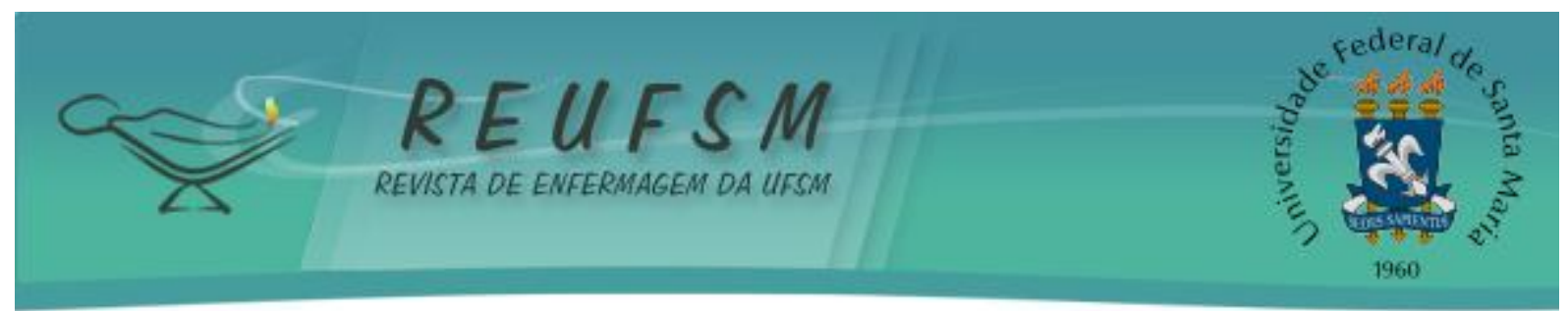

Dessa maneira, torna-se essencial a implementação de escalas para avaliação da dor em RNs, as quais são consideradas ferramentas clínicas de baixo custo e de alto impacto na identificação desse sintoma. ${ }^{2}$

Com relação a percepção do enfermeiro sobre o conhecimento e aprimoramento, os depoimentos a seguir apontam o uso benéfico da escala:

[...] conhecimento e aprimoramento que se consegue para desenvolver e avaliar com eficácia a dor. (Brilhante)

[...] achei bom para fazermos o treinamento dela [escala] e depois podermos utilizá-la [...] (Topázio)

Em virtude disso, nota-se que os enfermeiros valorizaram a proposta do estudo, a saber, o exercício de se utilizar uma escala para avaliação da dor. Assim, acredita-se que a educação continuada, promovida por meio de treinamentos e capacitações, seja o alicerce para a busca e inserção de conhecimentos novos ou pouco difundidos na prática assistencial de enfermagem, tal como o da avaliação da dor nos RNs prematuros. Dessa maneira, os enfermeiros necessitam buscar por aprimoramento e aperfeiçoamento de suas práticas e processo de trabalho, a fim de promover um cuidado integral e qualificado.

A avaliação foi citada pelos participantes, como um facilitador presente na utilização do instrumento, conforme demonstrado abaixo:

[...] comparações de um dia para o outro que na escala determinado dia estava com um nível de dor, no outro, com outro nível [...] um dia estava com mais dor, no outro dia ele estava pior [...] apareceu mais complicações [...] (Rubi)

[...] a dor influencia bastante no desenvolvimento deles, se tu souber avaliar e minimizar a dor do prematuro este vai ter uma recuperação mais rápida e eficiente. (Pérola)

[...] ter dados que se pode avaliar e não ficar no empírico. (Brilhante)

[...] identificar um desconforto que as vezes não era dor [...] (Topázio)

Percebe-se que os enfermeiros apontaram dentre outras vantagens da utilização da escala, a avaliação concreta e fidedigna da dor do RN, com possibilidades de acompanhar a evolução do quadro álgico com o passar dos dias. E ainda mencionaram conhecimento relacionado à influência da dor no desenvolvimento da criança.

Diante disso, é descrito que o efeito fisiológico da dor tem consequências importantes que aumentam a morbidade e a mortalidade dos prematuros. ${ }^{7}$ Com o intuito de minimizar esses agravos, apresenta-se como relevante uma atenção mais centrada na avaliação dos sinais e características que apontam a dor no RN prematuro, buscando métodos e intervenções para o seu alívio, a fim de melhorar sua qualidade de vida. ${ }^{15}$

Acredita-se que por meio da instrumentalização do conhecimento sobre a dor e o uso de escalas para mensurá-la, esse processo tornar-se-ia mais objetivo e científico, não sendo verificado apenas por meio da observação empírica. Os prematuros internados em UTIN costumam apresentar respostas comportamentais bastante diminuídas frente aos 


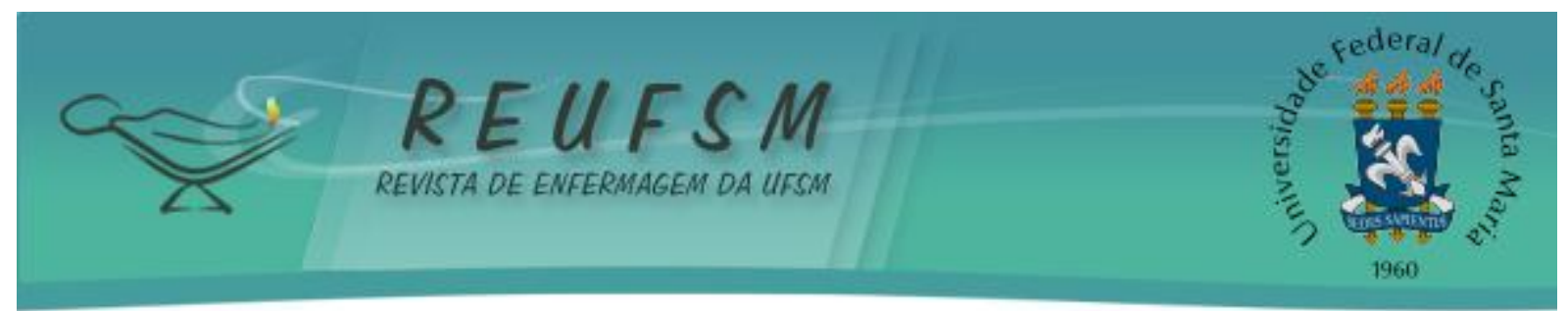

estímulos dolorosos, todavia elas poderiam ser identificadas de um modo mais adequado mediante a utilização de métodos científicos, como uma escala. Deste modo, seria mais fácil avaliar a evolução do quadro clínico, consequentemente evitando ou minimizando os agravos à sua saúde.

Diretamente relacionada com a avaliação da dor, a analgesia também foi citada como um benefício na utilização da escala, como explícito nesta fala:

[...] a gente não fica no "achômetro", no empírico [...] observa a necessidade de analgesia [...] dentro dos parâmetros préestabelecidos. (Brilhante)

Como citado anteriormente, constata-se, cada vez mais, a pertinência de preparo e conhecimento científico para a utilização das escalas de avaliação da dor, visando otimizar os benefícios que ela proporciona ao RN. Na prática, observa-se que algumas medicações prescritas para o alívio da dor, nas unidades de terapia intensiva, ficam restritas ao uso "somente quando necessário" deixando margem a precedentes, sejam na administração sem indicação precisa ou uso de outras medidas alternativas que não promovam a analgesia.

Além da administração de medicamentos, após uma correta avaliação da dor ou até mesmo na realização de procedimentos invasivos e dolorosos, sabe-se que existem medidas não farmacológicas para o alívio da mesma. Para tanto, em um estudo foi descrito que na presença de procedimentos dolorosos em uma Unidade Neonatal, há possibilidade de adoção de intervenções não medicamentosas por profissionais da enfermagem, incluindo medidas ambientais e comportamentais. ${ }^{13}$

Diante disso, mostra-se imprescindível que os enfermeiros estejam aptos a decodificar a "linguagem" do neonato, através da avaliação de suas mudanças comportamentais e fisiológicas, entre outras que apontem a presença da dor, a partir da utilização de escalas específicas.

Dessa maneira, na atualidade existem escalas que contribuem com os profissionais da saúde na avaliação do estímulo doloroso, as quais permitem a aferição de parâmetros fisiológicos e comportamentais, tanto isolados, quanto associados, auxiliando na determinação de intervenções específicas. ${ }^{14}$

Frente às facilidades e/ou benefícios da utilização destas escalas, a participação da equipe teve destaque, como revelado nesta explanação:

[...] principalmente para os funcionários [...] as gurias (técnicas de enfermagem) queriam saber o que eu estava fazendo, então explicava para elas também reconhecerem a dor neles. (Topázio)

Constata-se, nessa fala, que a equipe de enfermagem estava atenta ao processo de trabalho do enfermeiro, mediante a utilização da escala para avaliação da dor nos RNs prematuros. Quando essas práticas e tecnologias se referem ao manejo da dor no recémnascido, torna-se um desafio, que requer treinamento e capacitação, que devem ser transmitidos a toda equipe de enfermagem.

Assim sendo, a atuação da enfermagem é de grande relevância no que diz respeito ao cuidado dos neonatos. E o tratamento da dor alude na adoção de atitudes multimodais, uma vez que diversos fatores podem ser responsáveis pela sua ocorrência. ${ }^{16}$

Dessa maneira, a avaliação e o manejo da dor no RN prematuro devem ser foco do cuidado integral da enfermagem, além desses profissionais necessitarem considerar esse evento como um sinal vital de relevância clínica. ${ }^{17}$ 


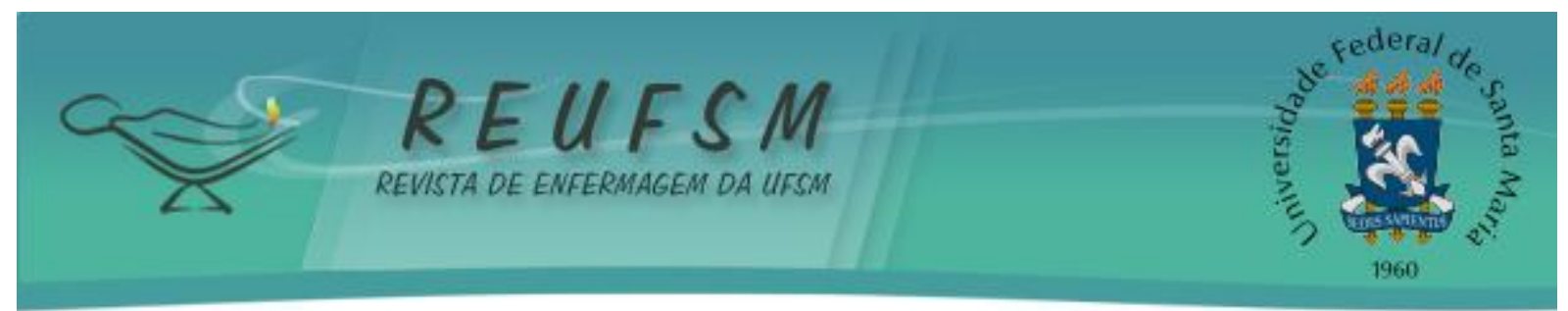

Diante de todas as facilidades e/ou benefícios apontadas pelos participantes, observa-se a real necessidade de formação e desenvolvimento dos profissionais de enfermagem, para a utilização das escalas de avaliação da dor. Neste contexto, leva-se em consideração a importância de se conhecerem os métodos de avaliação, sinais e características que são expressos pelos prematuros e definem a dor, sua fisiologia, bem como a farmacologia e medidas alternativas para o seu alívio e/ou inibição.

Assim sendo, salienta-se a participação e o trabalho em equipe da enfermagem no cuidado à saúde dos RNs prematuros, como um fator imprescindível na qualidade da assistência prestada nas UTIN.

A subcategoria dificuldades vivenciadas pelos enfermeiros na aplicação de um instrumento para avaliação da dor nos RNs prematuros de uma UTIN, ressalta alguns elementos presentes no cotidiano do trabalho da enfermagem. Este depoimento aponta para a sua aplicação e momento adequado, como exposto:

Sim, eu tive dificuldade, alguns dias não tive tempo de poder avaliar [...] qual seria o melhor momento para mim aplicar a tabela. (Pérola)

A avaliação da dor deve ser considerada o quinto sinal vital. Assim sendo, poderia ser incorporada à rotina de verificação dos sinais vitais, com o intuito de manter uma avaliação constante e periódica. Desta maneira, seriam aplicadas intervenções para o manejo da dor, de acordo com as necessidades de cada um. Salienta-se que tais respostas não podem ser utilizadas isoladamente para a indicação de dor, visto que se deve levar em consideração que o êxito da percepção ou valorização da dor no neonato exige a capacidade de avaliação de quem está aplicando o instrumento. ${ }^{7}$

Também relacionado com a aplicação, observou-se que a falta dessa rotina na unidade também é considerada uma dificuldade vivenciada pelo profissional.

A única dificuldade foi fazer diariamente, porque não é rotina na unidade [...] acabava esquecendo [...] mas não que eu ache difícil de ser aplicado ou inviável. (Rubi)

Em relação às rotinas e normas estabelecidas em uma unidade, entende-se que ambas podem contribuir para a qualidade do cuidado prestado, porém elas só podem ser efetivas se o profissional se colocar como norteador destes elementos de trabalho.

Em uma pesquisa desenvolvida com enfermeiros durante um Simpósio Internacional de Cuidados Intensivos Pediátricos e Neonatais em São Paulo foi encontrado que a maioria dos participantes não realizavam a avaliação da dor em sua rotina de trabalho, sendo que esta também não era considerada o quinto sinal vital. ${ }^{4}$

No que tange à utilização do instrumento para avaliação da dor, houve unanimidade por parte dos enfermeiros que afirmaram positivamente o desejo de implementar as escalas apresentadas nesta pesquisa, nos locais onde trabalhavam.

Por sua vez, mostra-se visível, na rotina diária dos cuidados prestados aos RNs prematuros nas UTIN uma diversidade de procedimentos invasivos e dolorosos para que seja possível a manutenção das suas vidas. Assim sendo, torna-se de extrema relevância a adoção de medidas relacionadas à avaliação e tratamento da dor em neonatos, uma vez que estes são submetidos a cuidados de extrema complexidade e elevado grau doloroso. Sem dúvida tratar a dor de forma apropriada relaciona-se com a redução de complicações e a diminuição da morbimortalidade. 


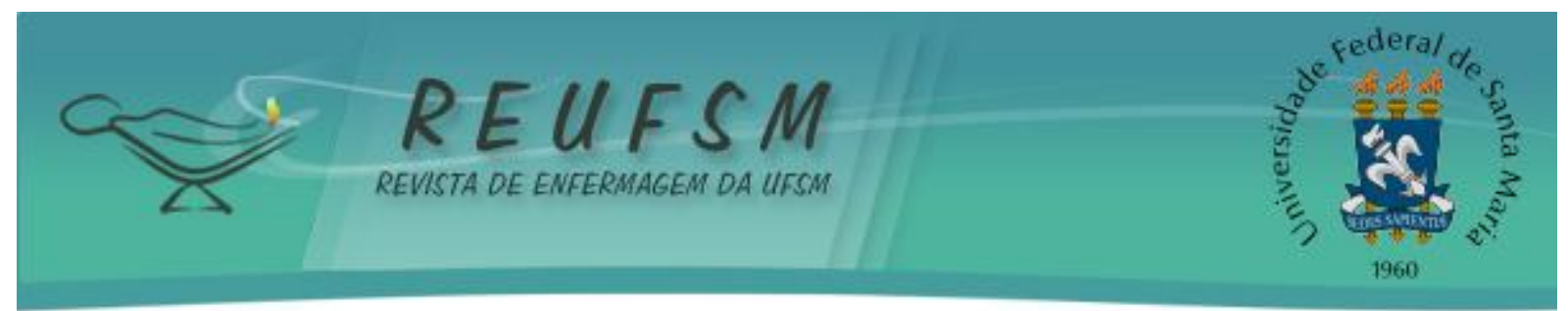

Nesse sentido, para uma avaliação da dor em neonatos, torna-se imprescindível a utilização de instrumentos, os quais necessitam ser aprendidos, implementados e utilizados continuamente, de modo a garantir um tratamento seguro e eficaz. ${ }^{18}$

Diante deste cenário, torna-se essencial o trabalho do enfermeiro, profissional que necessita de preparo e de experiência para conduzir a assistência de enfermagem baseada em procedimentos técnico-científicos que garantam a qualidade desta, além de planejar o cuidado ao RN, para que a equipe desempenhe de modo eficiente o cuidado. Sendo assim, se faz necessário um instrumento que normatize esta conduta. ${ }^{13}$

\section{CONCLUSÃO}

Este estudo buscou observar a percepção dos enfermeiros em relação à utilização de uma escala para avaliação da dor do recém-nascido prematuro, na tentativa de fundamentar a prática da atuação da enfermagem diante deste fenômeno, evidenciando sua identificação e avaliação, para posterior garantia de um atendimento singular e humano.

Neste contexto, foi possível visualizar que os profissionais desconhecem, cientificamente, as novas tecnologias utilizadas para o manejo da dor nos RN's prematuros, embora estas sejam de fundamental importância para o desenvolvimento efetivo destas crianças que necessitam de tantos cuidados especiais. Inquestionavelmente a dor acarreta diversos agravos à saúde, a médio ou longo prazo.

Contudo, nota-se que os enfermeiros, participantes deste estudo, estão abertos ao novo e comprometidos com a humanização da assistência. Este fato foi identificado na aceitação de todos os participantes pela inserção desta nova tecnologia dentro da UTIN, da referida instituição.

Assim sendo, o desafio para a enfermagem não é a percepção e o entendimento acerca da dor, já que os participantes, mesmo que de forma empírica, acabam por reconhecê-la nos neonatos. Acredita- se que a capacitação e o desenvolvimento da equipe de enfermagem, para uma atuação eficiente frente ao manejo da dor, consistem na principal estratégia para o aprimoramento do processo de trabalho nas UTIN, na busca por um cuidado integral e uma enfermagem consciente do seu papel na minimização e inibição dos fatores condicionantes da dor.

\section{REFERÊNCIAS}

1. Brasil. Ministério da Saúde. Atenção à saúde do recém-nascido: guia para os profissionais de saúde. Brasília (DF): Ministério da Saúde; 2012. (Cuidados com o recém-nascido prétermo; 4).

2. Santos LM, Pereira MP, Santos LFN, Santana RCB. Avaliação da dor no recém- nascido prematuro em Unidade de Terapia Intensiva. Rev Bras Enferm [Internet]. 2012 jan/fev [acesso em 2014 jun 12];65(1):27-33. Disponível em: http://www.scielo.br/pdf/reben/v65n1/04.pdf. doi: http://dx.doi.org/10.1590/S003471672012000100004.

3. Brasil. Ministério da Saúde. Atenção à Saúde do recém-nascido: guia para os profissionais de saúde. Brasília (DF): Ministério da Saúde; 2013. (Intervenções comuns, icterícia e infecções; 2).

4. Santos MZ, Kusahara DM, Pedreira MLG. Vivências de enfermeiros intensivistas na avaliação e intervenção para alívio da dor na criança. Rev Esc Enferm USP [Internet]. 2012 out [acesso em 2014 jun 13];46(5):1074-81. Disponível em: http://www.scielo.br/pdf/reeusp/v46n5/en_06.pdf. doi: http://dx.doi.org/10.1590/S008062342012000500006. 


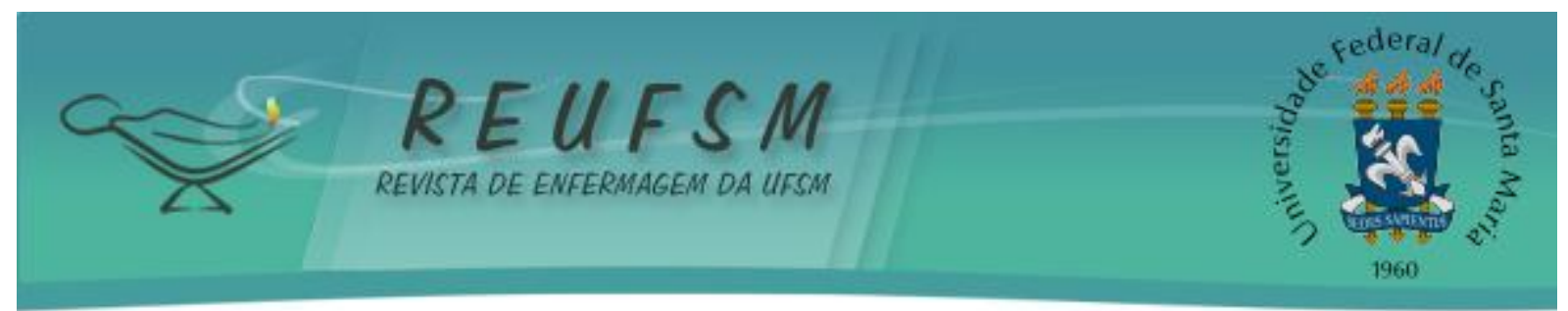

5. Vinall J, Grunau RE. Impact of repeated procedural pain-related stress in infants born very preterm. Pediatr Res [Internet]. 2014 maio [acesso em 2014 dez 21];75(5):584-7. Disponível em: http://www.ncbi.nlm.nih.gov/pmc/articles/PMC3992189/

6. Martins SW, Dias FS, Enumo SRF, Paula KMP. Avaliação e controle da dor por enfermeiras de uma unidade de terapia intensiva neonatal. Rev Dor [Internet]. 2013 jan/mar [acesso em 2014 jun 13];14(1):21-6. Disponível em: http://www.scielo.br/pdf/rdor/v14n1/v14n1a06.pdf. doi: http://dx.doi.org/10.1590/S1806-00132013000100006.

7. Tamez RN. Intervenções no cuidado neuropsicomotor do prematuro UTI neonatal. Rio de Janeiro: Guanabara Koogan; 2009.

8. Bueno M, Costa P, Oliveira AAS, Cardoso R, Kimura AF. Translation and adaptation of the premature infant pain profile into Brazilian Portuguese. Texto \& Contexto Enferm [Internet]. 2013 jan/mar [acesso em 2014 dez 18];22(1):29-35. Disponível em: http://www.scielo.br/pdf/tce/v22n1/04.pdf.

9. Bottega FH, Fontana RT. A dor como quinto sinal vital: utilização da escala de avaliação por enfermeiros de um hospital geral. Texto \& Contexto Enferm [Internet]. $2010 \mathrm{abr} / \mathrm{jun}$ [acesso em 2014 jul 10];19(2):283-90. Disponível em: http://www.scielo.br/pdf/tce/v19n2/09. doi: http://dx.doi.org/10.1590/S0104-0 7072010000200009.

10. Bueno M, Kimura AF, Diniz CSG. Evidências científicas no controle da dor no período neonatal. Acta Paul Enferm [Internet]. 2009 nov/dez [acesso em 2014 dez 18];22(6):82832. Disponível em: http://www.scielo.br/pdf/ape/v22n6/a16v22n6.pdf.

11. Brasil. Ministério da Saúde. Conselho Nacional de Saúde. Resolução $n^{\circ} 466$, de 12 de dezembro de 2012. Diretrizes e normas regulamentadoras de pesquisa envolvendo seres humanos [Internet]. Brasília; 2012 [acesso em 2014 dez 13]. Disponível em: http://conselho.saude.gov.br/resolucoes/2012/Reso466.pdf.

12. Minayo MCS. O desafio do conhecimento: pesquisa qualitativa em saúde. $11^{\mathrm{a}}$ ed. São Paulo: Hucitec; 2010.

13. Aquino FM, Christoffel MM. Dor neonatal: medidas não-farmacológicas utilizadas pela equipe de enfermagem. Rev RENE [Internet]. 2010 [acesso em 2014 mar 4];11(N Esp):16977. Disponível

em:

http://www.revistarene.ufc.br/revista/index.php/revista/article/viewFile/483/pdf.

14. Presbytero R, Costa MLV, Santos RCS. Os enfermeiros da unidade neonatal frente ao recémnascido com dor. Rev RENE [Internet]. 2010 jan/mar [acesso em 2014 jul 10];11(1):125-32. Disponível em: http://www.revistarene.ufc.br/revista/index.php/revista/article/view/355/pdf.

15. Silva TM, Chaves EMC, Cardoso MVLML. Dor sofrida pelo recém-nascido durante a punção arterial. Esc Anna Nery Rev Enferm [Internet]. 2009 out/dez [acesso em 2010 mar 12];13(4):726-32. Disponível em: http://www.scielo.br/pdf/ean/v13n4/v13n4a06.pdf. doi: http://dx.doi.org/10.1590/S1414-81452009000400006.

16. Carvalho CG, Carvalho VL. Manejo clínico da enfermagem no alívio da dor em neonatos. e-Scientia [Internet]. 2012 [acesso em 2014 jul 10];5(1):23-30. Disponível em: http://revistas.unibh.br/index.php/dcbas/article/view/199/464.

17. Santos LM, Silva TPCC, Santana RCB, Matos KKC. Sinais sugestivos de dor durante a punção venosa periférica em prematuros. Rev Enferm UFSM [Internet]. 2012 jan/abr 


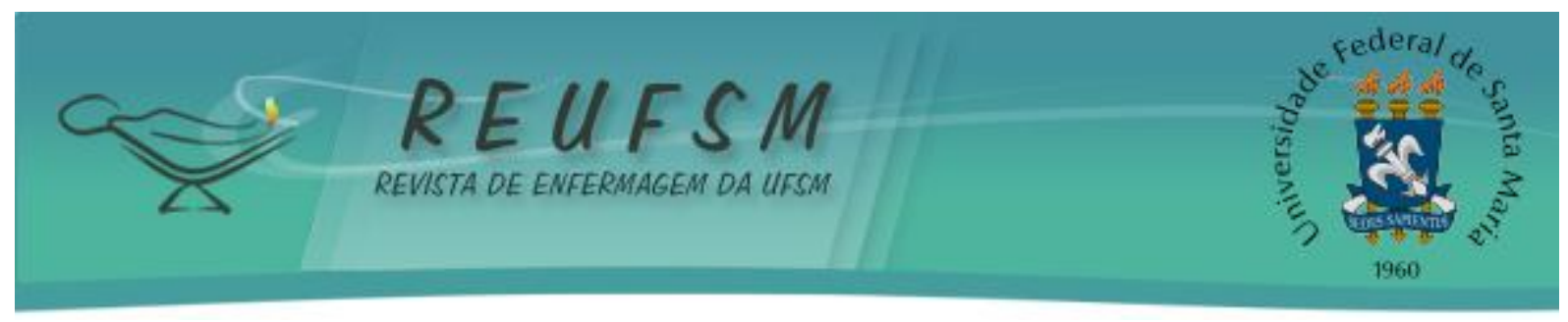

[acesso em 2014 ago 13];2(1):1-9. Disponível em: http://cascavel.ufsm.br/revistas/ojs2.2.2/index.php/reufsm/article/view/3510/3121.

18. Maxwell LG, Malavolta CP, Fraga MV. Assessment of pain in the neonate. Clin Perinatol [Internet]. 2013 Sept [acesso em 2014 dez 16];40(3):457-69. Disponível em: http://www.sciencedirect.com/science/article/pii/S0095510813000560.

Data de recebimento: 03/08/2014

Data de aceite: 23/01/2015

Contato com autor responsável: Xênia Martins Monfrim. Endereço: Rua Visconde de Jaguari NN217. CEP: 96010530, Pelotas, Rio Grande do Sul, Brasil.

E-mail: xenia.monfrim@bol.com.br 